\title{
Leaf Whorl Inoculation Method for Screening Sugarcane Rust Resistance
}

\author{
Sushma G. Sood, Jack C. Comstock, and Neil C. Glynn, United States Department of Agriculture-Agricultural \\ Research Service, Sugarcane Field Station, Canal Point, FL 33438
}

\begin{abstract}
Sood, S. G., Comstock, J. C., and Glynn, N. C. 2009. Leaf whorl inoculation method for screening sugarcane rust resistance. Plant Dis. 93:1335-1340.

Brown rust, caused by Puccinia melanocephala, and orange rust, caused by P. kuehnii, are agronomically important diseases of sugarcane in Florida. Cultivar resistance is the best means of controlling these diseases. Natural infection has been the primary means of assessing resistance in sugarcane cultivars against rusts; unfortunately, natural infection is not always efficient in identifying resistant cultivars due to variable environmental conditions. Therefore, a more reliable screening method is needed to effectively select resistant genotypes. An inoculation technique was evaluated for identification of brown and orange rust resistance in sugarcane cultivars. Inoculations were performed in the field by placing a $0.5-\mathrm{ml}$ urediniospore suspension in the leaf whorl of three individual sugarcane stalks per plant using a pipette. Symptoms developed on leaves of all the susceptible cultivars after 4 weeks, and appeared as a band of pustules. Plants were rated for their reaction to rust 4 weeks after inoculation. The optimum concentrations of inoculum for expression of brown and orange rust symptoms were determined. The most severe brown rust and orange rust symptoms were observed using inoculum containing $10^{5}$ and $10^{4}$ urediniospores $/ \mathrm{ml}$, respectively. Clones in several stages of the Canal Point breeding program were screened for their rust reaction by leaf whorl inoculation. The technique enabled rapid screening of a large number of cultivars in field plantings using a small amount of inoculum and limited man hours.
\end{abstract}

Brown rust of sugarcane, caused by Puccinia melanocephala Syd. \& P. Syd., was first recorded in the United States in 1978. It can cause substantial losses (6-8) and has been responsible for the withdrawal of commercial cultivars in the United States and in most sugarcanegrowing regions of the world. In 2007, a second rust of sugarcane, orange rust, caused by P. kuehnii (W. Krüger) E.J. Butler, was recorded in Florida for the first time (9). Orange rust has caused significant yield losses in Florida because the widely planted commercial cv. CP 80-1743 is susceptible to infection. Orange rust has now been confirmed in the Central American sugarcane industries of Costa Rica (4), Nicaragua (4), Guatemala (15), Mexico, and Panama (A. S. Guerra-Moreno, unpublished data).

The frequent applications of fungicide and low net profit preclude their use for controlling sugarcane brown rust (14) and orange rust (19), making cultivar resistance and cultural practices the most viable alternatives. Cultivars developed in the Canal Point breeding program not only are

Corresponding author: S. Sood

E-mail: sushma.sood@ars.usda.gov

Accepted for publication 21 August 2009.

doi:10.1094/PDIS-93-12-1335

This article is in the public domain and not copyrightable. It may be freely reprinted with customary crediting of the source. The American Phytopathological Society, 2009. used in the United States, but also many Central American sugarcane industries use them for either breeding or commercial production. This means that an effective method for screening genotype reactions to rust pathogens is an important priority. Natural infection has been the primary means of assessing rust resistance in sugarcane cultivars. Although natural infection is useful in assessing resistance, it has several disadvantages. For rating brown rust reactions in Florida, sugarcane plants should be 3 to 7 months old during the months of April to late May, when the environment is conducive for rust development. Usually, commercial plantings meet these conditions but not all plants in the cultivar development program are at this age during these months. Sugarcane seedlings are transplanted to the field in May and, often, minimal brown rust develops on these plants. Some cultivars in the early stages of the Canal Point breeding program that were rated as rust resistant after natural infection developed moderate to severe brown rust just prior to release or just after release.

Races of brown rust were first proposed to exist in Florida during the 1980s $(10,17)$, a finding that was later confirmed by Shine et al. (18). However, only a single race was thought to exist in Australia at the time of one study (20). Brown rust natural infection relies on the race of brown rust pathogen present or prevalent in the fields where the plants are being evaluated. Because there is no simple means of identify- ing rust races, there is no knowledge of which race or races the plants are actually being exposed to at a specific time.

The advantages of artificial inoculation include uniform exposure of all plants under disease-favorable conditions to a sufficient concentration of pathogen inoculum containing urediniospores collected from different locations and different cultivars (to collect possible different races of brown rust) to induce disease in susceptible genotypes. Sugarcane plants can be inoculated by spraying plants with a spore suspension. However, this method needs favorable ambient conditions (high humidity, requiring a dew chamber) for rust disease development. It requires a large amount of inoculum, which is a limitation in the case of obligate fungi, such as orange and brown rusts of sugarcane, and cannot be used to screen thousands of clones in the cultivar development program. Also, inoculation of sugarcane plants by spraying is not possible in the field, especially in Florida, where frequent rain showers occur that could wash off urediniospores from the leaves. Inoculation methods for glasshouse screening of resistance have been developed for pathogens of various plants but all have their shortcomings, and the lack of a suitable glasshouse screening technique for sugarcane rusts remains. Screening of cultivar resistance by detached sugarcane leaf segment inoculation (1) is not feasible for a large number of cultivars and may not fully reflect results with whole plants in the field and glasshouse (13). Bugdee and Sappenfield (3) suggested that the success of screening cultivars of cotton resistant to Fusarium spp. is dependent on the development of a rapid technique that eliminates escapes and ensures an accurate expression of resistance. They concluded that screening by inoculation must give reproducible wilt reactions that correspond with known resistance among cotton cultivars and correlate with field observations under natural conditions. These same principles apply to screening sugarcane rust reactions by inoculation.

In this study, we sought to develop a technique that will accurately screen large numbers of cultivars for orange and brown rust resistance under field conditions. A leaf whorl inoculation method for screening orange and brown rust resistance in sugarcane was developed and evaluated.

\section{MATERIALS AND METHODS}

Urediniospore collection, storage, germination, and suspension. Rust ured- 
iniospores were collected by vacuuming the abaxial side of symptomatic leaves. Brown rust urediniospores were collected from leaves of susceptible cultivars from several locations (to collect possible different races of brown rust) and mixed together in equal amounts. Orange rust urediniospores were harvested from infected leaves of cv. CP 80-1743, collected from different locations the day before inoculation. Brown and orange rust urediniospores can be stored for a week at room temperature without losing any viability. For the long term, urediniospores can be stored at $-70^{\circ} \mathrm{C}$. Storage of orange rust urediniospores at $-70^{\circ} \mathrm{C}$ required heat shock $\left(42^{\circ} \mathrm{C}\right.$ for $2.5 \mathrm{~min}$ ) before use (2).

Urediniospore germination was determined as described by Braithwaite (2). Freshly harvested brown rust urediniospore germination in water was $58 \%$ and the addition of $0.001,0.002,0.004,0.008$, or $0.016 \%$ (vol/vol) 1-nonanol or Tween20 was not able to increase brown rust urediniospore germination. Freshly harvested orange rust urediniospores germinated without the germination stimulator 1-nonanol (Sigma-Aldrich, St. Louis); however, addition of $0.002 \%$ 1-nonanol increased urediniospore germination from 45 to $75 \%$. Addition of $0.001,0.002$, $0.004,0.008$, or $0.016 \%$ ( $\mathrm{vol} / \mathrm{vol}$ ) Tween20 was not able to increase orange rust urediniospore germination. Germination of urediniospores of both rusts stored at room temperature for a week was the same as that of freshly harvested urediniospores.

To obtain a uniform spore suspension, brown and orange rust urediniospores were separately suspended in $2 \mathrm{ml}$ of sterile, distilled water containing $0.1 \%$ ( $\mathrm{vol} / \mathrm{vol}$ ) Tween-20 (Sigma-Aldrich) and were mixed by vortex. Sterile distilled water was added to brown rust urediniospores and sterile distilled water containing $0.002 \%$ 1-nonanol (vol/vol; 2) was added to orange rust urediniospore suspensions to obtain the desired inoculum concentration.

Inoculum concentration optimization. The optimum concentrations of brown and orange rust inocula were determined in the field. To optimize inoculum concentration of brown rust, five susceptible cultivars (CPCL 02-6061, CPCL 02-6063, CPCL
02-7626, CPCL 02-7643, and CPCL 05$1360)$ and two resistant cultivars (CP 801743 and CP 00-1101) for brown rust and CP 80-1743, susceptible for orange rust, were selected. Due to the recent introduction of orange rust in Florida, cultivars with long-term resistance to orange rust by natural infection have not been identified.

Five inoculum concentrations $\left(10^{2}, 10^{3}\right.$, $10^{4}, 10^{5}$, and $10^{6}$ urediniospores $/ \mathrm{ml}$ ) were used to optimize inoculum concentration. Inoculum was prepared as described above. Inoculum concentration was determined by hemacytometer (Bausch and Lomb, Rochester, NY) and germination of urediniospores was determined prior to every inoculation trial as described by Braithwaite (2). Brown rust urediniospore germination was $58 \%$ and orange rust urediniospore germination was $75 \%$ after addition of $0.002 \%$ ( vol $/ \mathrm{vol})$ 1-nonanol. When urediniospore germination was less than described earlier, inoculum concentration was adjusted according to germination. Plants to be inoculated were marked by cutting off one-third of the tips of the uppermost leaves so they could easily be identified at the time of rating. A $0.5-\mathrm{ml}$ aliquot of urediniospore suspension containing $10^{2}, 10^{3}, 10^{4}, 10^{5}$, or $10^{6}$ urediniospores $/ \mathrm{ml}$ was placed separately inside the leaf whorls of three individual stalks per replicate using a repeater pipette (model 8100; Nichiryo, Tokyo). Care was taken not to disturb the plants after inoculation so that all the inoculum would remain in the whorl. There were four replicates and experiments were repeated once for a total of two trials. Plant age at the time of inoculation was 4 months; the field was planted in November and inoculated in late March during the morning hours. During inoculation and the symptom development period, daily mean low and high temperatures ranged from 16 to $30^{\circ} \mathrm{C}$, relative humidity was between 47 and $91 \%$, and wind speed was 2 to $30 \mathrm{kph}$.

Symptoms appeared on leaves (of susceptible cultivars) as a band of pustules after they emerged from the whorl. Disease assessment started 1 week after inoculation and continued at weekly interval for 4 weeks. Disease reactions for both rust pathogens were rated on a 0 -to-4 scale,

Table 1. Brown rust (Puccinia melanocephala) and orange rust (P. kuehnii) ratings of sugarcane cultivars in different stages of the cultivar development program after leaf whorl inoculation in 2008

\begin{tabular}{lccccccc}
\hline & \multicolumn{5}{c}{ Percentage of cultivars $^{\mathbf{z}}$} \\
\cline { 2 - 4 } Rating & Stage II & Stage III & Stages IIIi-IV & & Stage II & Stage III & Stages IIIi-IV \\
\cline { 2 - 5 } \cline { 6 - 8 } & $9 \mathrm{C}$ & $22 \mathrm{~B}$ & $35 \mathrm{~A}$ & & $40 \mathrm{~A}$ & $20 \mathrm{~B}$ & $13 \mathrm{C}$ \\
1 & $42 \mathrm{~B}$ & $40 \mathrm{~B}$ & $47 \mathrm{~A}$ & & $29 \mathrm{~B}$ & $36 \mathrm{~A}$ & $15 \mathrm{C}$ \\
2 & $28 \mathrm{~A}$ & $24 \mathrm{~A}$ & $12 \mathrm{~B}$ & & $24 \mathrm{~B}$ & $26 \mathrm{~B}$ & $58 \mathrm{~A}$ \\
3 & $13 \mathrm{~A}$ & $10 \mathrm{~A}$ & $5 \mathrm{~B}$ & & $6 \mathrm{~B}$ & $14 \mathrm{~A}$ & $12 \mathrm{~A}$ \\
4 & $8 \mathrm{~A}$ & $4 \mathrm{~B}$ & $2 \mathrm{C}$ & & $1 \mathrm{~B}$ & $4 \mathrm{~A}$ & $2 \mathrm{~B}$ \\
\hline
\end{tabular}

${ }^{\mathrm{z}}$ All stages have different cultivars. Means of stage II, stage III, and stage III increase and stage IV (Stages IIIi-IV) followed by the same letter are not statistically significantly different $(P=0.05)$ based on Fisher's protected least significant difference test. where $0=$ no symptoms, $1=$ chlorotic flecks, 2 = orange or red-brown lesions, 3 $=$ one to five pustules with sporulation (production of urediniospores), and $4=$ five or more pustules with sporulation that coalesced, causing the area of leaves to become necrotic. Rust ratings of 0 and 1 were considered resistant, 2 was considered moderately resistant, and 3 and 4 were considered susceptible. The inoculum concentration that produced rust symptoms receiving the significantly $(P=0.05)$ higher rating was considered the optimum for use in further studies. Inoculum concentrations of $10^{2}, 10^{3}, 10^{4}, 10^{5}$, and $10^{6}$ urediniospores $/ \mathrm{ml}$ were transformed to $\log$ $2,3,4,5$, and 6 urediniospores $/ \mathrm{ml}$, respectively. Standard errors for means were determined by the SAS software package (version 9.1; SAS Institute Inc., Cary, NC).

Screening of cultivars for rust resistance by leaf whorl inoculation. The cultivars in the second clonal selection stage (Stage II), third clonal selection stage (Stage III), first ratoon of third clonal selection stage(Stage III increase), and fourth clonal selection stage (Stage IV) of the Canal Point cultivar development program were screened for brown rust resistance in 2007 and 2008 and for orange rust resistance in 2008. There were four replicates in Stage III increase and Stage IV, two replicates in Stage III, and the Stage II test was not replicated. In 2007, 1,359 Stage II cultivars, 135 Stage III cultivars, 43 Stage III increase cultivars, and 18 Stage IV cultivars were tested for brown rust resistance. In 2008, 1,273 Stage II cultivars, 134 Stage III cultivars, 46 Stage III increase cultivars, and 17 Stage IV cultivars were tested for both orange and brown rust resistance. Inoculations were performed using the optimum spore concentration as described earlier: $10^{5}$ for brown rust and $10^{4}$ for orange rust. Brown rust and orange rust inoculation studies were done in separate trials. Plant age at the time of inoculation varied between 4 and 7 months depending on the stage within the Canal Point breeding program; fields were planted from late November to late December and inoculated in late March through early June during the morning hours. During inoculation and the symptom development period, daily mean low and high temperatures ranged from 16 to $30^{\circ} \mathrm{C}$, relative humidity was between 47 and $91 \%$, and wind speed was 2 to $30 \mathrm{kph}$. Plants were rated for their reaction to rust on the above-mentioned rating scale 4 weeks after inoculation. The percentage of cultivars that showed different rust ratings was determined and results are summarized in Table 1. Experimental observation showed that cultivars have variable rust reaction to brown and orange rusts. Therefore, the rust reaction data from these screenings was consolidated, and cultivars were separated according to their brown and orange rusts ratings in the following 
categories: resistant to both rusts, moderately resistant to both rusts, susceptible to both rusts, resistant to brown rust and susceptible to orange rust, resistant to orange rust and susceptible to brown rust, moderately resistant to brown rust and resistant to orange rust, moderately resistant to orange rust and resistant to brown rust, moderately resistant to brown rust and susceptible to orange rust, and moderately resistant to orange rusts and susceptible to brown rust. Cultivars in Stage III increase and Stage IV were combined together and statistical analyses were conducted using the GLM procedure with SAS. Means were separated using Fisher's protected least significant difference at $P=0.05$.

Comparison between leaf whorl inoculation and natural infection. To determine the efficiency of the whorl inoculation technique, brown rust ratings of sugarcane cultivars after natural infection were compared with brown rust ratings of the same cultivars after whorl inoculation. In 2007, 135 cultivars in Stage III, 43 cultivars in Stage III increase, and 18 cultivars in Stage IV were rated for their brown rust reaction after whorl inoculation and after natural infection. These stages were chosen for the study because they were replicated. The rust rating scale for natural infection was the same as for the leaf whorl inoculation. The data were analyzed by the REG procedure with SAS. This trial was not repeated in 2008 because some cultivars were susceptible to both brown and orange rusts, and brown rust ratings after natural infection could not be determined accurately.

Comparison between rust ratings of 2007 and 2008 leaf whorl inoculation. To determine reproducibility of the whorl inoculation technique, brown rust ratings of cultivars in 2008 were compared with brown rust ratings of the same cultivars in 2007. Cultivars in 2008 Stage III clones were advanced from 2007 Stage II. Similarly, 2008 Stage III increase and Stage IV were advanced from 2007 Stage III. These cultivars were rated for their brown rust reaction in 2007 as well as in 2008. Brown rust ratings of 134 cultivars in Stage III, 46 cultivars in Stage III increase, and 17 cultivars in Stage IV obtained for each cultivar were pooled within year and compared between years (2007 and 2008) by the REG procedure with SAS.

\section{RESULTS}

Inoculum concentration optimization. Disease assessments after leaf whorl inoculation of sugarcane cultivars showed that some cultivars had sporulating pustules as soon as the leaves emerged from the whorl. Some cultivars had pustules with sporulation after 2 weeks of inoculation but all susceptible cultivars had sporulating pustules after 4 weeks of inoculation. There was no change in rust rating after 4 weeks (results not shown). There- fore, in all other experiments, rust symptoms of sugarcane cultivars were rated 4 weeks after inoculation. The optimum inoculum concentration for brown rust leaf whorl inoculation was determined on five susceptible cultivars (CPCL 02-6061, CPCL 02-6063, CPCL 02-7626, CPCL 027643, and CPCL 05-1360) and two resistant cultivars (CP 80-1743 and CP 001101) (Fig. 1). All susceptible cultivars showed significantly $(P=0.05)$ higher brown rust ratings when inoculated with $10^{5}$ urediniospores $/ \mathrm{ml}$ than the brown rust ratings when inoculated with $10^{2}, 10^{3}$, and $10^{4}$ urediniospores $/ \mathrm{ml}$; however, increasing concentration from $10^{5}$ to $10^{6}$ urediniospores $/ \mathrm{ml}$ resulted in no significant $(P=$ $0.05)$ increase in rust ratings. The brownrust-resistant cultivars showed no reaction when inoculated with $10^{1}$ and $10^{3}$ urediniospores $/ \mathrm{ml}$. These cultivars showed no reaction or few chlorotic flecks when inoculated with $10^{4}$ to $10^{6}$ urediniospores $/ \mathrm{ml}$ (Fig. 1). CP 80-1743 showed significantly $(P=0.05)$ higher orange rust susceptibility ratings when inoculated with $10^{4}$ urediniospores/ml than the rust ratings when inoculated with $10^{2}$ and $10^{3}$ urediniospores $/ \mathrm{ml}$ and there was no significant $(P=0.05)$ difference between rust rating with $10^{4}$, $10^{5}$, and $10^{6}$ urediniospores/ml (Fig. 2).

Screening of cultivars for rust resistance. Different percentages of cultivars in different stages of the cultivar development program showed different rust ratings for brown and orange rusts (Table 1). A significantly $(P=0.05)$ higher percentage of cultivars showed resistant (0) brown rust ratings in Stage III increase and Stage IV than in Stage III and Stage II, whereas significantly $(P=0.05)$ more cultivars were susceptible to brown rust in Stage II than Stage III, Stage III increase, and Stage IV. In contrast, Stage II had significantly $(P=0.05)$ more cultivars resistant to orange rust than other stages. Stage III increase and Stage IV had significantly $(P$ $=0.05$ ) fewer orange-rust-resistant cultivars than other stages. Stage III had significantly $(P=0.05)$ more orange-rustsusceptible cultivars than other stages. Stage III increase and Stage IV had significantly $(P=0.05)$ fewer cultivars with moderate resistance to brown rust than Stage II and Stage III. In contrast, Stage III increase and Stage IV had significantly $(P$ $=0.05$ ) more cultivars with moderate resistance to orange rust than Stage II and Stage III (Table 1).

Cultivars were separated into different categories according to their brown and orange rust ratings obtained by leaf whorl inoculation (Table 2). Rust screening data showed that $51 \%$ of Stage II, $34 \%$ of Stage III, and $25 \%$ of Stage III increase and Stage IV cultivars were resistant to both orange and brown rusts (Table 2). A significantly $(P=0.05)$ greater percentage of cultivars in Stage III increase and Stage IV were moderate to both rusts than in Stage

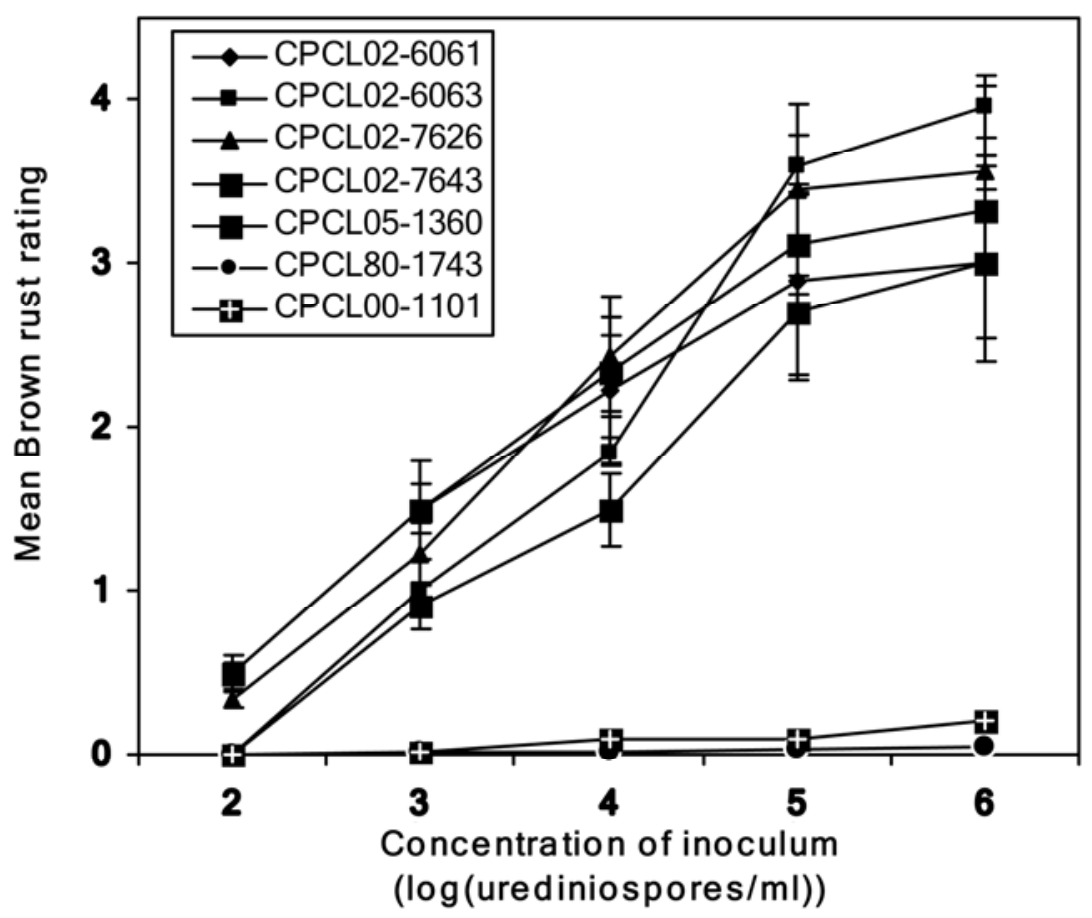

Fig. 1. Mean brown rust (Puccinia melanocephala) ratings in five susceptible and two resistant sugarcane (Saccharum spp.) cultivars after leaf whorl inoculation using five inoculum concentrations. Inoculum concentrations of $10^{2}, 10^{3}, 10^{4}, 10^{5}$, and $10^{6}$ urediniospores/ml were transformed to $\log 1,2,3$, 4, 5, and 6, respectively. Each data point stands for mean of two trials, each with four replications of three plants each, and bars represent standard errors. The rust symptoms were rated on a 0-to- 4 scale, where $0=$ no symptoms, $1=$ chlorotic flecks, $2=$ red-brown lesions, $3=$ one to five pustules with sporulation (production of urediniospores), and $4=$ five or more pustules with sporulation that coalesce, causing the area of leaves to become necrotic. 
II and Stage III. Similarly, a significantly $(P=0.05)$ greater percentage of cultivars in Stage III increase and Stage IV were susceptible to both rusts, moderately resistant to orange rust and resistant to brown rust, and moderately resistant to orange rust and susceptible to brown rust than in Stage II and Stage III.

Comparison between leaf whorl inoculation and natural infection. Ratings for brown rust recorded after leaf whorl inoculation of cultivars in Stage IV, Stage III increase, and Stage III were compared with rust ratings obtained after natural infection for the same cultivars. The brown rust ratings obtained for different stages were combined and relationship was determined (Fig. 3). Ratings showed a sig- nificant $(P=0.05)$ correlation $\left(r^{2}=0.72\right)$ between brown rust ratings after leaf whorl inoculation and natural infection. However, $10 \%$ of cultivars that had a susceptible rating (rating 3 and 4) after leaf whorl inoculation did not show a susceptible reaction after natural infection and none of the cultivars that had a susceptible rating (by natural infection) showed a resistant reaction after leaf whorl inoculation (Fig. 3).

Comparison between rust ratings of 2007 and 2008 leaf whorl inoculation. Selected sugarcane cultivars from 2007 Stage III were advanced to 2008 Stage IV and Stage III increase. Similarly, selected sugarcane cultivars from 2007 Stage II were advanced to 2008 Stage III. The

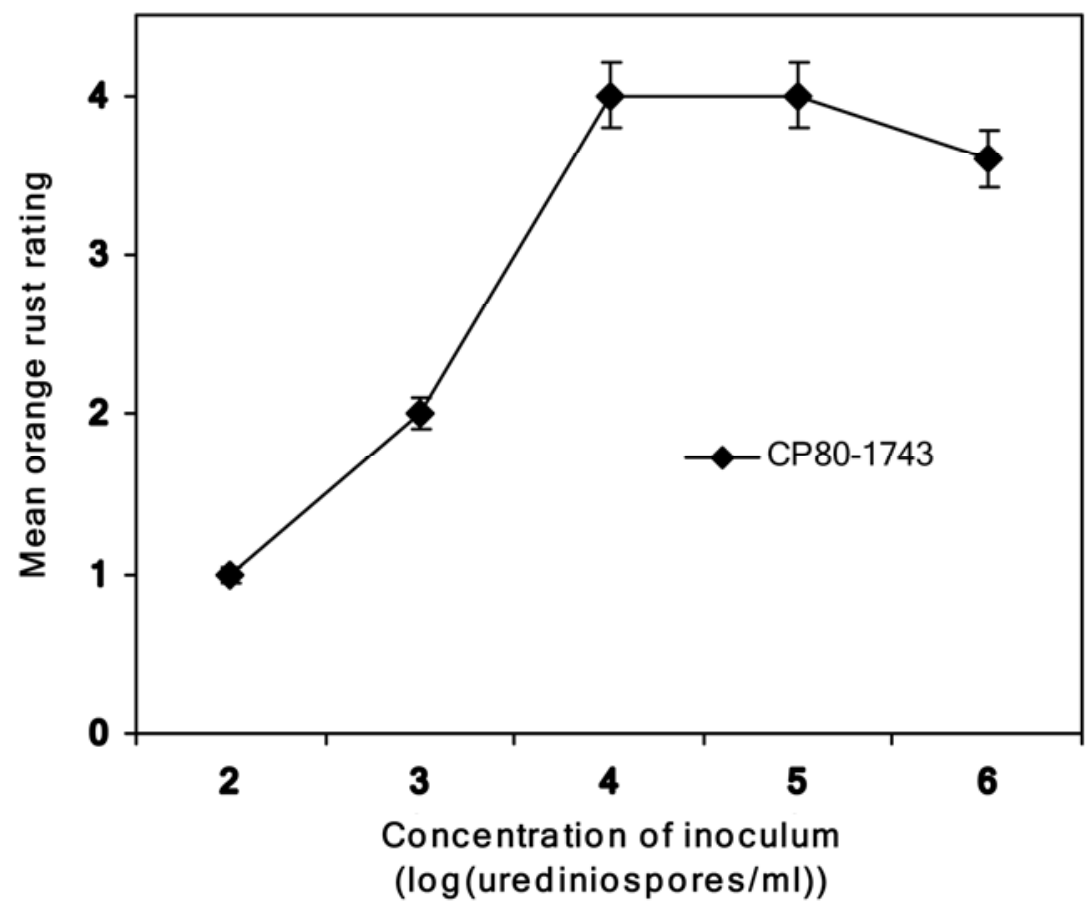

Fig. 2. Mean orange rust (Puccinia kuehnii) ratings of sugarcane (Saccharum spp.) cv. CP 80-1743 after leaf whorl inoculation using five inoculum concentrations. Inoculum concentrations of $10^{2}, 10^{3}$, $10^{4}, 10^{5}$, and $10^{6}$ urediniospores/ml were transformed to $\log 1,2,3,4,5$, and 6 , respectively. Each data point stands for the mean of two trials, each with four replications of three plants each, and bars represent standard errors. The rust symptoms were rated on a 0 -to- 4 scale, where $0=$ no symptoms, $1=$ chlorotic flecks, 2 = red-brown lesions, $3=$ one to five pustules with sporulation (production of urediniospores), and $4=$ five or more pustules with sporulation that coalesce, causing the area of leaves to become necrotic. brown rust disease data obtained for each cultivar were pooled within year and compared between years (2007 and 2008) using correlation analysis. There was a significant $(P=0.05)$ correlation $\left(r^{2}=0.84\right)$ between brown rust resistance ratings in 2007 and in 2008 (Fig. 4).

\section{DISCUSSION}

Increase in rust susceptibility ratings with increase in brown rust and orange rust inoculum concentration from $10^{2}$ to $10^{5}$ and $10^{2}$ to $10^{4}$ urediniospores $/ \mathrm{ml}$, respectively, and no increase in susceptibility ratings with further increase in inoculum concentration are consistent with other studies. Petersen (16) also observed an increase in pustule production with increasing density of urediniospores of $P$. graminis f. sp. tritici and found that the total number of pustules produced reached a plateau when spore density reached $4 \times$ $10^{4}$ spores $/ \mathrm{ml}$. With leek rust ( $P$. allii), the severity was reduced when low densities of inoculum (5) were used. Gilles and Kennedy (11) also observed an increase in rust pustule production with increasing inoculum density. They also found that a higher spore concentration was required to successfully infect leek leaves at temperatures higher than $20^{\circ} \mathrm{C}$.

The percentages of cultivars resistant to both rusts and the individual rusts varied between the stages. In general, more cultivars in Stage III increase and Stage IV were resistant to brown rust than to orange rust. The reason for brown rust variation may be, in part, because of previous selection for brown rust resistance using natural infection. All the cultivars in Stage III, Stage III increase, and Stage IV had been rated for their brown rust reaction and obviously susceptible clones were eliminated. There was no previous screening for orange rust resistance because the incursion of orange disease had only occurred in 2007. The higher percentage of orangerust-susceptible cultivars in Stage II was most likely due to chance. The proportion of resistant cultivars at each stage serves as a baseline for the level of resistance. Leaf whorl inoculation screening data have not been used for cultivars selection for differ-

Table 2. Percent cultivars assigned to different categories according to their ratings for brown rust (Puccinia melanocephala) and orange rust (P. kuehnii) after leaf whorl inoculation in 2008

\begin{tabular}{|c|c|c|c|}
\hline \multirow[b]{2}{*}{ Category } & \multicolumn{3}{|c|}{ Percentage of cultivars ${ }^{\mathrm{z}}$} \\
\hline & Stage II & Stage III & Stages IIIi-IV \\
\hline Resistant to both rusts & $51 \mathrm{~A}$ & $34 \mathrm{~B}$ & $25 \mathrm{C}$ \\
\hline Moderate to both rusts & $5 \mathrm{~B}$ & $7 \mathrm{~B}$ & $13 \mathrm{~A}$ \\
\hline Susceptible to both rusts & $2 \mathrm{~B}$ & $4 \mathrm{~A}$ & $3 \mathrm{~A}$ \\
\hline Resistant to brown rust and susceptible to orange rust & $4 \mathrm{C}$ & $12 \mathrm{~A}$ & $8 \mathrm{~B}$ \\
\hline Resistant to orange rust and susceptible to brown rust & $5 \mathrm{~A}$ & $5 \mathrm{~A}$ & $2 \mathrm{~B}$ \\
\hline Moderately resistant to brown rust and resistant to orange rust & $13 \mathrm{~B}$ & $17 \mathrm{~A}$ & $2 \mathrm{C}$ \\
\hline Moderately resistant to orange rust and resistant to brown rust & $16 \mathrm{~B}$ & $18 \mathrm{~B}$ & $40 \mathrm{~A}$ \\
\hline Moderately resistant to brown rust and susceptible to orange rust & $2 \mathrm{~A}$ & $2 \mathrm{~A}$ & $2 \mathrm{~A}$ \\
\hline Moderately resistant to orange rust and susceptible to brown rust & $2 \mathrm{~B}$ & $1 \mathrm{~B}$ & $5 \mathrm{~A}$ \\
\hline
\end{tabular}

\footnotetext{
${ }^{\mathrm{z}}$ All stages have different cultivars. Means of stage II, stage III, and stage III increase and stage IV (Stages IIIi-IV) followed by the same letter are not statis-
} tically significantly different $(P=0.05)$ based on Fisher's protected least significant difference test. 
ent stages in 2008 because this study was not completed at that time. Once the selection of resistant sugarcane cultivars by leaf whorl inoculation is in use for several years, the elimination of susceptible clones in the early stages should result in a higher proportion of resistant clones in the later stages.

Significant $(P=0.05)$ correlation $\left(r^{2}=\right.$ 0.72 ) between brown rust ratings after whorl inoculation and after natural infection show that whorl inoculation efficiently screens sugarcane cultivars for rust resistance with fewer escapes. However, $10 \%$ of cultivars that had a susceptible rating (rating 3 and 4) after leaf whorl inoculation did not show a susceptible reaction after natural infection and could be the escapes under natural infection whereas, in leaf whorl inoculations, rust urediniospores introduced into the leaf whorl were in constant contact with the host under disease-conducive conditions for infection and had sufficient time to germinate and colonize the host, which resulted in fewer escapes. Leaf wetness and temperature influence brown rust development in sugarcane (6). Dry conditions could negatively affect brown and orange rust development but, once urediniospores are inside the leaf whorls after inoculation, they were less exposed to exterior drying environmental conditions. Leaf whorls may have acted as a barrier, reducing the effects of temperature fluctuation, increasing the availability of moisture, and creating a favorable microenvironment for urediniospores to germinate and infect. All the clones susceptible to natural infection showed susceptible reactions after leaf whorl inoculation. The rust urediniospore concentrations used for leaf whorl inoculation of brown and orange rusts may be capable of overwhelming moderately resistant cultivars and capable of distinguishing between less susceptible and moderately resistant cultivars. Goulter et al. (12) reported that higher inoculum density of $P$. helianthi shortened generation time and produced more rust pustules on leaves of sunflower. They also suggested that severe rust infection of hybrid sunflower during 1980 and 1981 in Queensland, Australia could be due to the presence of higher spore density rather than pathogenic variation (12).

Significant $(P=0.05)$ relationships between brown rust ratings of cultivars in all the stages of the cultivar development program with whorl inoculation obtained in 2007 and 2008 show that leaf whorl inoculation is reproducible and dependable. These results demonstrate that proper whorl inoculation of sugarcane cultivars with brown rust urediniospores would result in consistent infection.

The results presented in this study show that the leaf whorl inoculation technique is very useful, reproducible, and efficient for screening a large number of sugarcane cultivars for $P$. melanocephala and $P$. kuehnii resistance in field conditions.

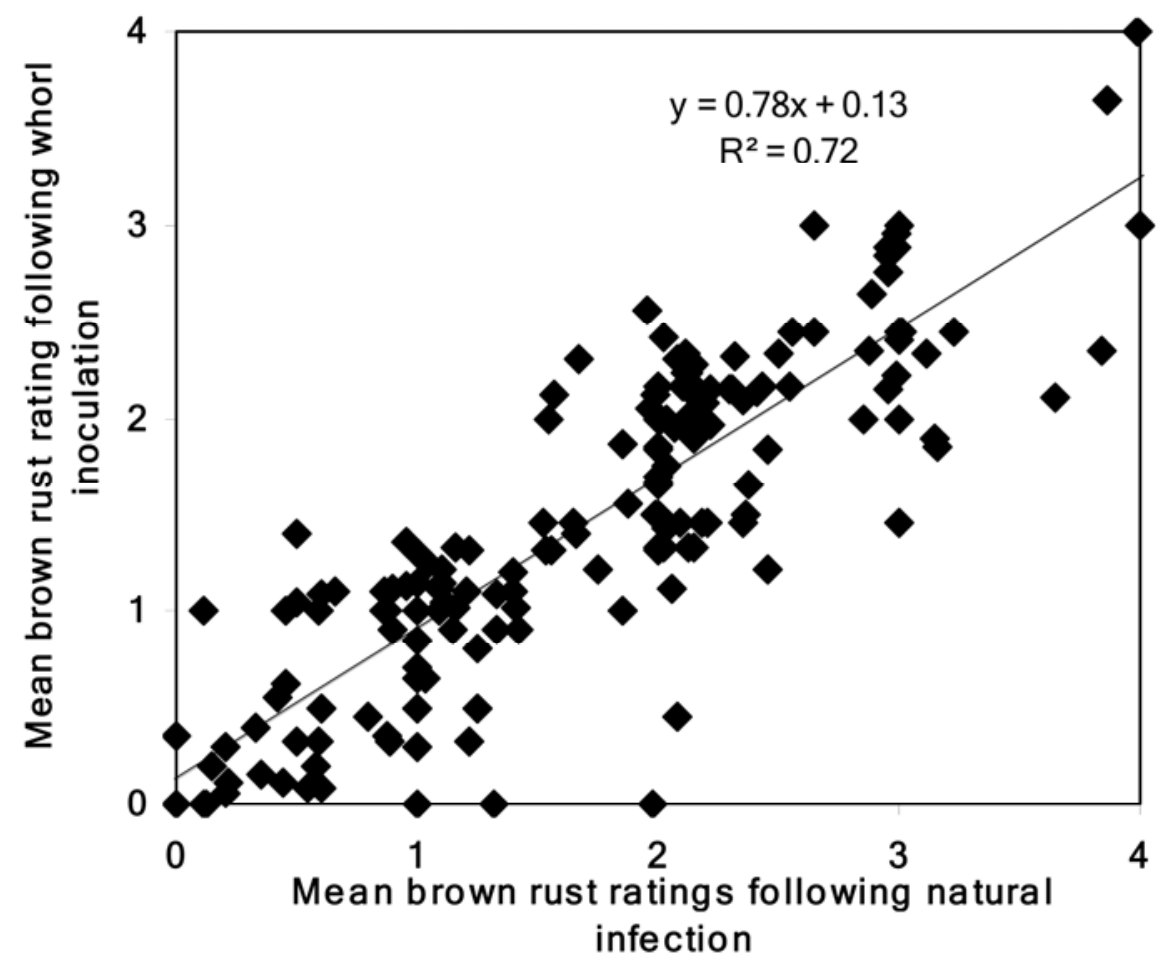

Fig. 3. Comparison between brown rust (Puccinia melanocephala) ratings of 2007 stage IV, stage III increase, and stage III sugarcane (Saccharum spp.) cultivars after leaf whorl inoculation and after natural infection. The rust symptoms were rated on a 0 -to-4 scale, where $0=$ no symptoms, $1=$ chlorotic flecks, 2 = red-brown lesions, 3 = one to five pustules with sporulation (production of urediniospores), and $4=$ five or more pustules with sporulation that coalesce, causing the area of leaves to become necrotic.

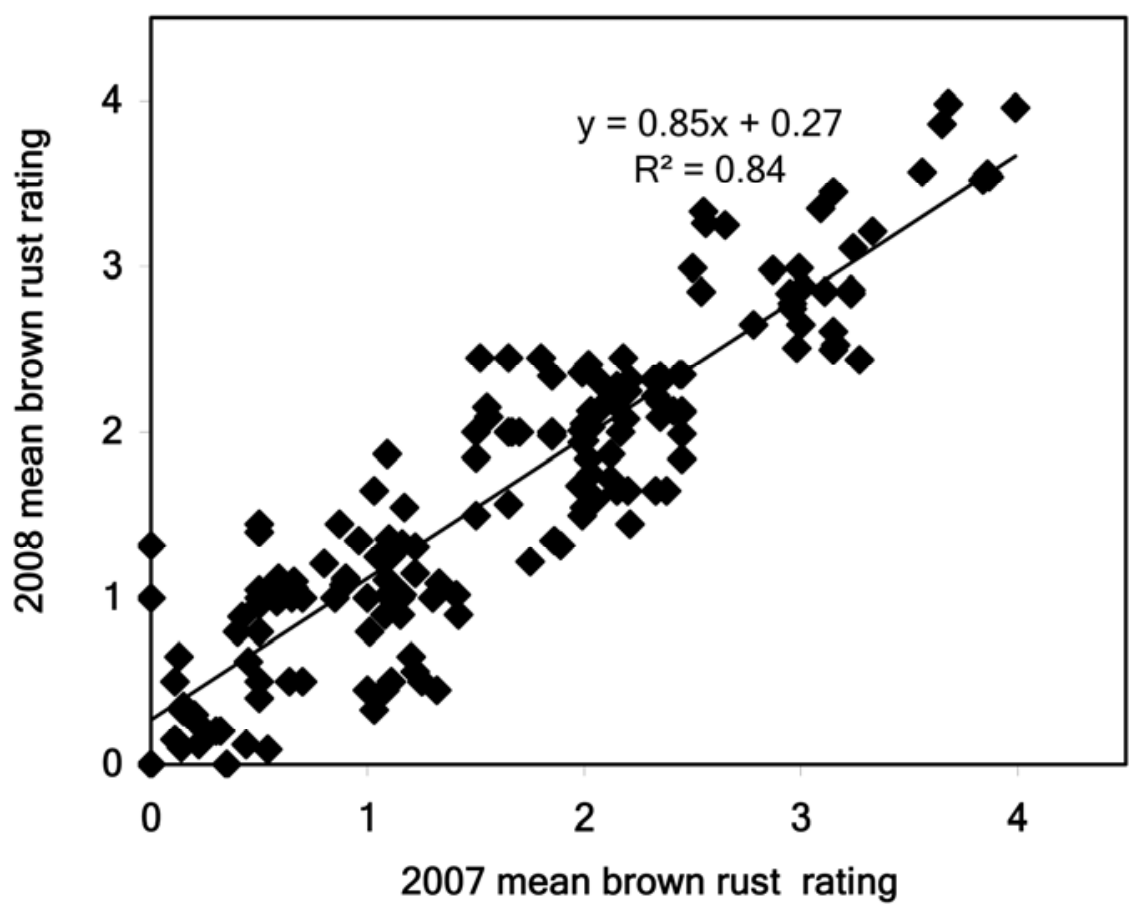

Fig. 4. Comparison between brown rust (Puccinia melanocephala) ratings after leaf whorl inoculation of 2008 stage IV, stage III increase, and stage III cultivars (that were advanced from 2007 stage III increase, stage III, and stage II, respectively) and brown rust ratings of the same sugarcane (Saccharum spp.) cultivars collected in 2007 . The rust symptoms were rated on a 0 -to- 4 scale, where $0=$ no symptoms, 1 = chlorotic flecks, 2 = red-brown lesions, 3 = one to five pustules with sporulation (production of urediniospores), and $4=$ five or more pustules with sporulation that coalesce, causing the area of leaves to become necrotic. 


\section{LITERATURE CITED}

1. Asnaghi, C., D’Hont, A., Glaszmann, J.-C., and Rott, P. 2001. Resistance of sugarcane cultivar R 570 to P. melanocephala isolates from different geographic locations. Plant Dis. 85:282-286.

2. Braithwaite, K. S. 2005. Assessing the impact that pathogen variation has on the sugarcane breeding program. SRDC Final Report for Project BSS258. BSES Publ. SD05018.

3. Bugdee, W. M., and Sappenfield, W. P. 1967. Varietal reactions of cotton after stem and root inoculation with Fusarium oxysporum f. sp. vasinfectum. Phytopathology 58:212-214.

4. Chavarría, E., Sabiros, F., Vega, J., Ralda, G., Glynn, N. C., Comstock, J. C., and Castlebury, L. A. 2009. First report of Puccinia kuehnii, causal agent of orange rust of sugarcane in Costa Rica and Nicaragua. Plant Dis. 93:425.

5. Clarkson, J. P., Kennedy, R., Phelps, K., Davies, J., and Bowtell, J. 1997. Quantifying the effects of reduced doses of propiconazole (Tilt) and initial disease incidence on leek rust development. Plant Pathol. 46:952-963.

6. Comstock, J. C., and Raid, R. N. 1994. Sugarcane common rust. Pages 1-10 in: Current Trends in Sugarcane Pathology. K. S. Bhargava, G. P. Rao, A. G. Gillaspie, Jr., P. P. Upadhyaya, A. Bergamin Filino, V. P. Agni- hotri, and C. T. Chen, eds. International Books and Periodicals Supply Service, Delhi, India.

7. Comstock, J. C., Shine, J. M., Jr., and Raid, R. N. 1992. Effect of rust on sugarcane growth and biomass. Plant Dis. 76:175-177.

8. Comstock, J. C., Shine, J. M., Jr., and Raid, R. N. 1992. Effect of early rust infection on subsequent sugarcane growth. Sugar Cane 4:7-9.

9. Comstock, J. C., Sood, S. G., Glynn, N. C., Shine, J. M., Jr., McKemy, J. M., and Castlebury, L. A. 2008. First report of Puccinia kuehnii, causal agent of orange rust of sugarcane, in the United States and in the Western Hemisphere. Plant Dis. 92:175.

10. Dean, J. L., and Purdy, L. H. 1984. Races of the sugar cane rust fungus, Puccinia melanocephala, found in Florida. Sugar Cane 1:15-16.

11. Gilles, T., and Kennedy, R. 2003. Effects of an interaction between inoculum density and temperature on germination of Puccinia allii urediniospores and leek rust progress. Phytopathology 93:413-420.

12. Goulter, K. C., Kochman J. K., and Brown J. F. 1984. Investigations into the increased rust (Puccinia helianthi) intensity on some hybrid sunflower cultivars grown in Queensland. Aust. J. Agric. Res. 35:99-106.

13. Hsiang, T., Cook, S., and Zhao, Y. 2004. Studies on biology and control of daylily rust in
Canada. Daylily J. 59:47-57.

14. Jiang, D. K. 1985. Chemical control of sugarcane rust $P$. melanocephala. Rep. Taiwan Sugar Res. Inst. 108:25-34.

15. Ovalle, W., Comstock, J. C., Glynn, N. C., and Castlebury, L. A. 2008. First report of Puccinia kuehnii, causal agent of orange rust of sugarcane, in Guatemala. Plant Dis. 92:973.

16. Petersen, L. J. 1959. Relations between inoculum density and infection of wheat by urediniospores of Puccinia graminis var. tritici. Phytopathology 49:607-614.

17. Raid, R. N. 1989. Physiological specialization in sugarcane rust (Puccinia melanocephala) in Florida. Plant Dis. 73:183.

18. Shine, J. M., Jr., Comstock, J. C., and Dean, J. L. 2005. Comparison of five isolates of sugarcane rust and differential reaction on six sugarcane clones. Proc. Int. Soc. Sugar Cane Technol. 25:638-647.

19. Staier, T., Magarey, R., and Willcox, T. G. 2003. Control of orange rust in sugarcane with fungicides. Proc. Aust. Soc. Sugar Cane Technol. (CD ROM).

20. Taylor, P. W. J. 1992. Evidence for the existence of a single race of common rust caused by Puccinia melanocephala in Australian sugar cane cultivars. Aust. J. Agric. Res. 43:443-450. 